University of Nebraska - Lincoln

DigitalCommons@University of Nebraska - Lincoln

Faculty Publications, Department of Psychology

Psychology, Department of

2014

\title{
Rural Latino adolescent health: Preliminary examination of health risks and cultural correlates
}

\author{
Timothy D. Nelson \\ University of Nebraska-Lincoln, tnelson3@unl.edu \\ Katherine M. Kidwell \\ University of Nebraska-Lincoln \\ Brian E. Armenta \\ University of Nebraska-Lincoln, berikarmenta@gmail.com \\ Lisa J. Crockett \\ University of Nebraska-Lincoln, ecrockett1@unl.edu \\ Gustavo Carlo \\ University of Missouri, carlog@missouri.edu
}

See next page for additional authors

Follow this and additional works at: https://digitalcommons.unl.edu/psychfacpub

Part of the Agricultural and Resource Economics Commons, Applied Behavior Analysis Commons, Health Psychology Commons, Inequality and Stratification Commons, Medicine and Health Commons, Multicultural Psychology Commons, Place and Environment Commons, Race and Ethnicity Commons, and the Rural Sociology Commons

Nelson, Timothy D.; Kidwell, Katherine M.; Armenta, Brian E.; Crockett, Lisa J.; Carlo, Gustavo; and Whitbeck, Les B., "Rural Latino adolescent health: Preliminary examination of health risks and cultural correlates" (2014). Faculty Publications, Department of Psychology. 640.

https://digitalcommons.unl.edu/psychfacpub/640

This Article is brought to you for free and open access by the Psychology, Department of at DigitalCommons@University of Nebraska - Lincoln. It has been accepted for inclusion in Faculty Publications, Department of Psychology by an authorized administrator of DigitalCommons@University of Nebraska - Lincoln. 


\section{Authors}

Timothy D. Nelson, Katherine M. Kidwell, Brian E. Armenta, Lisa J. Crockett, Gustavo Carlo, and Les B. Whitbeck 


\title{
Rural Latino adolescent health: Preliminary examination of health risks and cultural correlates
}

\author{
Timothy D. Nelson,' Katherine M. Kidwell,' Brian E. Armenta,' \\ Lisa J. Crockett,' Gustavo Carlo, ${ }^{2}$ and Les B.Whitbeck ' \\ I. University of Nebraska-Lincoln, Lincoln, NE \\ 2. University of Missouri, Columbia, MO \\ Corresponding author - Timothy D Nelson, Department of Psychology, University of Nebraska-Lincoln, \\ 319 Burnett Hall, Lincoln, NE 68588-0308, USA; email tnelson3@unl.edu
}

\begin{abstract}
Latino adolescents living in rural settings may be at increased risk of health problems; however, data describing the health status of this population are limited. This study examined 60 rural Latino adolescents and found high rates of health risk, including at-risk/clinical results for hemoglobin AIC (23.3\%), high-density lipoprotein cholesterol (55\%), systolic blood pressure (21.7\%), and overweight/obesity (55\%). Time in sedentary behaviors was high and physical activity was limited. Adolescent language use was associated with health risk status, with greater use of English associated with lower risk. Health psychologists could promote improved health by providing health behavior interventions to this underserved population.
\end{abstract}

Keywords: adolescents, blood tests, health risk, Latino, rural

\section{Introduction}

The health status of Latino youth in the United States is an area of increasing research interest, especially in light of the rapid growth of this population (US Census Bureau, 2011) and findings of unique health risks among Latinos (Office of Minority Health, 2012; Schiller et al., 2012). Similarly, the physical health of youth in rural settings has been highlighted as an understudied area in need of research attention (Janicke and Davis, 2011). Despite increased re- search attention, studies focusing on youth who are both Latino and rural are limited. Most research with Latino youth has been in urban contexts, and such studies may not generalize to rural Latinos. Living in a rural setting carries unique health-related challenges (e.g. limited access to services; National Advisory Committee on Rural Health and Human Services (NACRHHS), 2008), and these challenges likely interact with the health challenges associated with Latino status, creating a population with po- 
tentially unique health risks. Given the increasing numbers of Latinos living in rural settings (US Census Bureau, 2011), and the paucity of research with this vulnerable population, data describing the health risks for rural Latino youth are clearly needed.

A growing literature has identified significant health risks for individuals from Latino backgrounds. Specifically, studies have shown increased risk among Latinos for prevalent and preventable conditions such as obesity and Type II diabetes (Office of Minority Health, 2012; Schiller et al., 2012; Zhang and Rodriguez- Monguio, 2012). Although Latinos have lower rates of heart disease than some other ethnic groups (Centers for Disease Control and Prevention (CDC), 2011; Schiller et al., 2012), they have relatively high rates of key risk factors, and heart disease is the leading cause of death in this population (US National Center for Health Statistics, 2010). Health risk is also reflected in critical health behaviors, with the majority of Latinos failing to meet recommendations for daily physical activity (Office of Minority Health, 2012; Schiller et al., 2012) and fruit/vegetable intake (Neumark-Sztainer et al., 1996). Promoting healthy behaviors could help in preventing and managing both chronic physical health problems and the mental health symptoms that often accompany these conditions. For example, Arredondo et al. (2012) found that vigorous physical activity was associated with lower levels of depressive symptoms among Latinos with hypertension. Furthermore, culture can have an important impact on health, and recent research suggests that certain health beliefs may contribute to health risks for Latino youth (Barroso et al., 2010).

Paralleling these ethnic health disparities is emerging evidence for the health challenges associated with rurality. On average, individuals living in rural settings have worse overall health and face greater obstacles to health-care access than urban residents (NACRHHS, 2008). Furthermore, there is some evidence that Latino ethnic status interacts with rural status to produce heightened health risk. Wickrama et al. (2007) examined data from the large-scale Ad-
dHealth Project and found that rurality was associated with a greater likelihood of having a chronic illness for Latino youth but not among White or African American youth. This study also found that certain cultural factors (e.g. language spoken at home) were associated with health risk, suggesting a complex interaction between culture, residential setting, and health for Latino youth.

Although large-scale studies of youth provide valuable information regarding the health status of rural Latino youth, they are often limited by their reliance on parent- or adolescentreport of health status. In addition to the potential bias associated with such reports, some important health risks (e.g. emerging cardiovascular issues) may be unknown to these reporters and can only be reliably identified through objective measures such as blood tests. Multimethod investigations of rural Latino health that include both objective measures of health status and parent/youth-report are lacking. Furthermore, limited data are available on the impact of key cultural variables (e.g. language status, ethnic identity) on the health status of rural Latino youth.

In light of the potential health risks faced by rural Latino youth, and the limitations of previous research, this article has two goals. First, we describe the health status of a sample of rural Latino adolescents, focusing on physical health status (particularly weight, Type II diabetes risk, and heart disease risk) and key health behaviors. Data are drawn from a multimethod assessment, including objective and interview data. Second, we provide a preliminary examination of the association between adolescent health status and cultural variables. Given the documented health risks associated with Latino status and rural residence, we hypothesized that youth in our sample would show high levels of health risks and that cultural variables would be associated with risk. The findings are expected to contribute to the literature by describing the objectively measured health status of a hard-to-reach population (i.e. rural Latinos from the Great Plains) and providing an examination of cultural correlates. This will 
be particularly relevant to health psychologists, who often provide culturally sensitive health promotion interventions targeting key health behaviors.

\section{Methods}

\section{Participants and procedures}

The participants included 60 Latino adolescents (age: $M=14.5$ years, standard deviation $(S D)=1.9$ years; $50 \%$ male) and their accompanying caregivers (age: $M=41.1$ years, $S D=$ 6.9 years; in the United States: $M=17.7$ years, $S D=7.8$ years; $78 \%$ female), living in one of the five rural communities in a Great Plains state. Participants were recruited via English and Spanish flyers (distributed by school staff and posted in locations such as restaurants and public libraries) to complete a singlesession study in their community (e.g. at a local school). Adolescents participated in both a medical screening and a face-to-face interview (45-60 minutes) with a trained interviewer, while caregivers participated in an interview only (45-60 minutes). Adolescent medical screenings were conducted by trained medical staff via a mobile medical unit and included assessments of height, weight, blood pressure, and a nonfasting blood sample. Adolescent and caregiver interviews were conducted by graduate and upper-level undergraduate students with previous interviewing experience. The interviewers each participated in a 2-hour training session and conducted two practice interviews prior to data collection. The interviews consisted of questions regarding demographics, family health history, health behavior, and culture-related issues. Standard rating scales were used (see "Measures" section), so no transcription or coding was needed. Interviews were conducted in either English or Spanish, depending on the preference of the respondent. Approximately two-thirds of the caregivers completed the interview in Spanish; over 90 percent of the adolescents completed it in English. Although all 60 adolescents completed the medical screening (including pro- viding a blood sample), 2 did not complete the interview due to a suspected developmental delay, resulting in a sample of 58 for interview measures. Participating caregivers received US\$20 and adolescents received a t-shirt. Caregivers were mailed a letter with the results of the adolescent's health screening. Each letter was written in both English and Spanish and included information on key health measures from the adolescent's blood test and physical assessment (see "Measures" section). For each measure, the adolescent's value, interpretation with relevant ranges, and basic recommendations (e.g. "Discuss results with your primary care provider") were provided. All procedures were approved by the Institutional Review Board of the University of Nebraska-Lincoln.

\section{Measures}

Blood test data. Adolescent blood samples were analyzed with a focus on risk factors for diabetes and heart disease. Specifically, standard tests for hemoglobin A1C (A1C; measure of diabetes status/risk; American Diabetes Association (ADA), 2010), C-reactive protein (CRP; measure of inflammation that has been linked to risk of heart disease; Ridker, 2003), triglycerides (indicator of fat in the blood; American Heart Association (AHA), 2012b), and cholesterol were conducted. A1C and high-sensitivity CRP tests can be interpreted from nonfasting data. Although triglycerides and cholesterol levels are often assessed using fasting blood tests, studies suggest that the results of these tests change minimally with food intake (Langsted et al., 2008) and that nonfasting results may be better predictors of long-term cardiac risk (Bansal et al., 2007). Our use of a nonfasting test was dictated primarily by feasibility concerns, but these studies indicate that the values obtained are clinically relevant. Clinical cutoffs from authoritative sources were used to categorize values, including A1C $(5.7 \%-6.4 \%$ - prediabetes; $\geq 6.5 \%$-diabetes; note: Interventions were recommended at $\geq 5.5 \%$, based on increases in risk at this level; ADA, 2010); CRP (>3.0 mg/L- 
high risk; Ridker, 2003); triglycerides (150-199 mg/dL - borderline high; 200-499 mg/dL high; $\geq 500 \mathrm{mg} / \mathrm{dL}$ - very high; AHA, 2012b); high-density lipoprotein (HDL) cholesterol $(<40$ $\mathrm{mg} / \mathrm{dL}-$ low for males; $<50 \mathrm{mg} / \mathrm{dL}-$ low for females; AHA, 2012b); low-density lipoprotein (LDL) cholesterol (130-159 mg/dL - borderline high; 160-189 mg/dL-high; $\geq 190 \mathrm{mg} / \mathrm{dL}-$ very high; AHA, 2012b).

Weight status and blood pressure. Using objectively measured height and weight data, adolescent body mass index (BMI)-for-age-percentiles were calculated and evaluated using CDC (2012) guidelines. Objectively measured adolescent blood pressure was converted to percentiles based on age and height, consistent with National High Blood Pressure Education Program (NHBPEP, 2004) criteria, with a focus on systolic blood pressure because this value has a stronger association with heart disease risk (American Heart Association (AHA), 2012a). Clinical cutoffs for prehypertension (90th-95th percentiles) and hypertension ( $>95$ th percentile) were used (NHBPEP, 2004).

Health behaviors. Key health behaviors were assessed during the adolescent interview. Using questions adapted from Ainsworth et al. (2000), adolescents reported the typical number of days per week they engaged in moderate and/or vigorous physical activity and the typical amount of time spent being physically active on those days, enabling the calculation of average minutes per day of physical activity. Sedentary behaviors were assessed by asking adolescents to report the amount of time spent each day watching television (TV), on the computer, and playing video games (adapted from Aaron et al., 1995). Finally, adolescents reported how many times they ate fruits and vegetables (reported separately) during the previous 7 days.

Cultural factors. To assess selected cultural factors potentially related to Latino health, participants answered questions regarding lan- guage use and ethnic identity. For language, both adolescents and their caregivers provided information about their own language use in different contexts (i.e. "In general, what language do you read in?"). For each question, participants responded on a 5-point scale $(1=$ Spanish only; 2 = Spanish more than English; $3=$ Both the same; 4 = English more than Spanish; and $5=$ English only). This 6-item scale (adapted from Marin et al., 1987) yields a mean language score with higher scores representing greater use of English and lower scores representing greater use of Spanish ( $\alpha=.79$ in this sample). Adolescent ethnic identity was measured using the 12item Multigroup Ethnic Identity Measure (Roberts et al., 1999; $\alpha=.84$ in this sample) assessing the degree to which one feels a sense of connection with, belonging to, and clarity regarding his/her ethnic background.

\section{Results}

Results indicated that a large percentage of the adolescents had blood test and physical exam values in the clinical or at-risk ranges. For A1C, 14 adolescents (23.3\%) met criteria for either "prediabetes" $(n=13)$ or "diabetes" $(n=1)$. However, using the ADA's (2010) cutoff of $5.5 \%$ for "initiating interventions," 33 adolescents (55\%) were above threshold. For triglycerides, 20 adolescents (33.3\%) were in either the "borderline high" or "high" ranges ( $n=10 ; 16.7 \%$, for each). Elevated CRP values were rare, with only 5 percent in the "highrisk" range. Similarly, only 3 adolescents (5\%) had LDL cholesterol levels in the "borderline high" or "high" ranges; however, 33 (55\%) were in the "low" range for HDL, which is associated with increased risk of heart disease. Over half of the sample was either "overweight" $(n=14 ; 23.3 \%)$ or "obese" $(n=20$; $33.3 \%$ ), and 21.7 percent had either "prehypertensive" $(n=4 ; 6.7 \%)$ or "hypertensive" $(n=9 ; 15 \%)$ systolic blood pressure readings. Overall, 60 percent $(n=36)$ had at least one clinical/at-risk blood value and 90 percent $(n$ = 54) had at least one clinical/at-risk blood/ physical exam value. 
Adolescent-reported health behaviors showed a similar pattern of high health risk. The mean daily physical activity was just 22.9 minutes, and approximately a quarter of the sample reported engaging in less than $10 \mathrm{~min}-$ utes of activity per day. Average time spent in sedentary behaviors was high, with adolescents reporting, on average, a total of approximately 4.5 hours per day watching TV (114.5 minutes/ day), on the computer (120.3 minutes/day), and playing video games (35.4 minutes/day). Fruit and vegetable consumption was low, with 65.5 percent eating vegetables less than once per day and 56.9 percent eating fruit less than once per day.

To examine the association between selected cultural variables and health risk factors, bivariate correlation analyses were conducted. Adolescent language use was significantly correlated with CRP $(r=-.26, p<.05)$ and systolic blood pressure $(r=-.26, p<.05)$ values, with greater use of English associated with lower (healthier) values. Adolescent language use did not correlate significantly with any of the other blood or physical exam measures. Caregiver language use did not correlate significantly with any of the health measures. To explore the association between cultural variables and overall health risks, we created a composite clinical health risk variable by calculating the number of blood and physical exam values that were in the at-risk/clinical range (possible range $=0-6$; actual range $=0-4$; mean $=1.57$ ). Adolescent language use was significantly associated with the number of at-risk/clinical values $(r=-.29, p<$ $.05)$, with greater use of English associated with fewer high-risk values. Adolescent ethnic identity was not significantly correlated with any of the health indicators.

\section{Discussion}

Overall, this study found alarmingly high rates of health risks in a sample of rural Latino adolescents, with 90 percent having at least one high-risk value related to diabetes and/ or heart disease. Unlike previous studies with this population, our study utilized objective measures (i.e. blood tests, physical exam measurements) of key health risk factors, avoiding common problems with underestimating health risks by relying on parent- or youth-report of health status. The results are consistent with the idea that Latino adolescents living in rural settings may be at particular risk for compromised health because of the double risk status of being Latino and rural. The elevated risk observed in this study highlights the importance of regular medical screening and evaluation for rural Latino youth and effective communication of these results to primary care pediatric providers.

Our results also point to the need for outreach and services from pediatric health psychologists focusing on facilitating healthy behavior change to reduce the long-term risks of Type II diabetes and heart disease in this population. For example, over half of the sample had an A1C level indicating the need to begin interventions (ADA, 2010). These interventions, which have the potential to lower long-term risk, consist largely of lifestyle modification efforts (e.g. increasing physical activity and healthy eating) commonly conducted by health psychologists. It may be important to combine culturally sensitive interventions that address the unique challenges of Latino status (e.g. potential issues related to ethnic identity, acculturation, or discrimination) as well as the unique challenges of rural status (e.g. potential issues with access to preventive services) to appropriately serve this population. Although such culturally tailored interventions are currently lacking, clinicians may seek to enhance existing protocols for lifestyle modification by providing information in both English and Spanish, conducting sessions in the client's preferred language, and identifying resources in the client's community and surrounding areas that could help promote healthy living (e.g. opportunities for health screening and education; locations for accessing healthy foods). Recent research suggests that communication between patients and medical providers may be especially important in promoting engagement and treatment adherence among Latinos (Villagran et al., 2012), so 
it is essential that providers present health recommendations in a clear and culturally appropriate manner. Along these lines, Mohan et al. (2013) suggested that illustrated diabetes management instructions may be useful with Latino patients as a supplement to traditional verbal or written directions, particularly for patients who speak limited English.

This study also found evidence for an association between adolescent language use and level of health risk. The finding that greater use of English was associated with lower risk should be interpreted with caution given the sample size; however, it is possible that greater comfort with English is protective for rural Latino adolescents because it facilitates better access to health information and services. Perhaps families who rely heavily on Spanish may be more hesitant to seek preventive and early intervention care because of concerns over possible language barriers. These barriers could further inhibit access in communities where access may already be an issue. Again, providing bilingual health services, and publicizing the availability of such services, may help to address this issue for families at greatest risk.

Some important limitations should be noted. The modest sample size limited our ability to examine culture-health associations using sophisticated analyses, and the representativeness of this sample is unknown. Our recruitment strategy of using a mobile health unit might have attracted families interested in obtaining a free health screening, possibly resulting in a sample less likely to be receiving regular medical care. Although we are cautious about generalizing to the broader rural Latino adolescent population, this study does provide insight into the health of youth who are likely to present to these types of outreach efforts, and the results make clear the high-risk status of these youth. This study was also limited by its one-time data collection, providing only a "snapshot" of this population. Future research should seek to access a larger sample and establish relationships that allow for repeated assessments over time. Despite these limitations, this study is the first to our knowl- edge to collect blood and physical exam data, as well as interview reports, from this difficult-to-access population. The results provide a valuable first look at health risks - and need for health behavior interventions-among rural Latino youth. Health psychologists, along with our health-care colleagues such as pediatricians, could be instrumental in addressing these issues by providing screening, health behavior psychoeducation, and behavioral lifestyle modification services to this chronically underserved population.

Funding - This work was funded by a grant from the University of Nebraska-Lincoln Office of Research.

\section{References}

Aaron, D. J., Kriska, A. M., Dearwater, S. R., et al. (1995) Reproducibility and validity of an epidemiologic questionnaire to assess past year physical activity in adolescents. American Journal of Epidemiology 142: 191-201.

Ainsworth, B. E., Bassett, D. R., Strath, A. M., et al. (2000) Comparison of three methods for measuring the time spent in physical activity. Medicine and Science in Sports and Exercise 32: S457-S464.

American Diabetes Association (ADA) (2010) Diagnosis and classification of diabetes mellitus. Diabetes Care 33 (Suppl. 1): S62-S69.

American Heart Association (AHA) (2012a) Understanding blood pressure readings. Accessed October 2, 2012 at http://www.heart.org/ HEARTORG/Conditions/HighBloodPressure/ AboutHighBloodPressure/Understanding-BloodPressure-Readings UCM 301764 Article.jsp

American Heart Association (AHA) (2012b) What your cholesterol levels mean. Accessed October 2, 2012 at http://www.heart.org/HEARTORG/ Conditions/Cholesterol/AboutCholesterol/What-Your-Cholesterol- Levels-Mean UCM_305562_Article.jsp

Arredondo, E. M., Mendelson, T., Elder, J. P., et al. (2012) The relation of medical conditions to depressive symptoms among Latinos: Leisure time physical activity as a moderator. Journal of Health Psychology 17(5): 742-752.

Bansal, S., Buring, J. E., Rifai, N., et al. (2007) Fasting compared with nonfasting triglycerides and risk 
of cardiovascular events in women. JAMA 298(3): 309-316.

Barroso, C. S., Peters, R. J., Johnson, R. J., et al. (2010) Belief and perceived norms concerning body image among African-American and Latino teenagers. Journal of Health Psychology 15(6): 858-870.

Centers for Disease Control and Prevention (CDC) (2011) Summary health statistics for U.S. adults 2010. Table 2. Accessed December 20, 2012 at http://www.cdc.gov/nchs/data/series/sr 10/ $\underline{\text { sr10_252.pdf }}$

Centers for Disease Control and Prevention (CDC) (2012) Basics about childhood obesity. Accessed October 2, 2012 at http://www.cdc.gov/obesity/ childhood/basics.html

Janicke, D. M. and Davis, A. M. (2011) Introduction to the special section: Rural health issues in pediatric psychology. Journal of Pediatric Psychology 36(6): 647-651.

Langsted, A., Freiberg, J. J., and Nordestgaard, B. G. (2008) Fasting and nonfasting lipid levels: Influence of normal food intake on lipids, lipoproteins, apolipoproteins, and cardiovascular risk prediction. Circulation 118: 2047-2056.

Marin, G., Sabogal, F., Marin, B. V., et al. (1987) Development of a short acculturation scale for Hispanics. Hispanic Journal of Behavioral Sciences 9(2): 183-205.

Mohan, A. V., Riley, M. B., Boyington, D. R., et al. (2013) Illustrated medication instructions as a strategy to improve medication management among Latinos: A qualitative analysis. Journal of Health Psychology 18(2): 187-197.

National Advisory Committee on Rural Health and Human Services (NACRHHS) (2008) The 2008 Report to the Secretary: Rural Health and Human Services Issues. Washington, DC: U.S. Department of Health and Human Services.

National High Blood Pressure Education Program (NHBPEP) (2004) The fourth report on the diagno- sis, evaluation, and treatment of high blood pressure in children and adolescents. Pediatrics 114(2): 555-576.

Neumark-Sztainer, D., Story, M., Resnick, M. D., et al. (1996) Correlates of inadequate fruit and vegetable consumption among adolescents. Preventive Medicine 25: 497-505.

Office of Minority Health (2012) Obesity and Hispanic Americans. Accessed December 20, 2012 at http://minorityhealth.hhs.gov/templates/content.aspx?ID $=6459$

Ridker, P. M. (2003) C-reactive protein. Circulation 108(12): e81-e85.

Roberts, R. E., Phinney, J. S., Masse, L. C., et al. (1999) The structure of ethnic identity of young adolescents from diverse ethnocultural groups. The Journal of Early Adolescence 19: 301-322.

Schiller, J. S., Lucas, J. W., Ward, B. W., et al. (2012) Summary health statistics for U.S. adults: National Health Interview Survey 2010. Vital and Health Statistics 10(252): 1-207.

US Census Bureau (2011) The Hispanic population: 2010. Accessed October 2, 2012 at http://www. census.gov/prod/cen2010/briefs/c2010br-04.pdf

US National Center for Health Statistics (2010) Deaths, final data for 2007. National Vital Statistics Reports 58(19): 1-136.

Villagran, M., Hajek, C., Zhao, X., et al. (2012) Communication and culture: Predictors of treatment adherence among Mexican immigrant patients. Journal of Health Psychology 17(3): 443-452.

Wickrama, K. A., Elder, G. H., and Abraham, W. T. (2007) Rurality and ethnicity in adolescent physical illness: Are children of the growing rural Latino population at excess health risk? The Journal of Rural Health 23(3): 228-237.

Zhang, H., and Rodriguez-Monguio, R. (2012) Racial disparities in the risk of developing obesity-related diseases: A cross-sectional study. Ethnicity $\mathcal{E}$ Disease 22(3): 308-316. 